\title{
Evaluación de la sostenibilidad de una agrocadena de suministro de producto fresco orientada al mercado internacional. Caso aplicado a la cadena del Aguacate Hass en el norte del Tolima
}

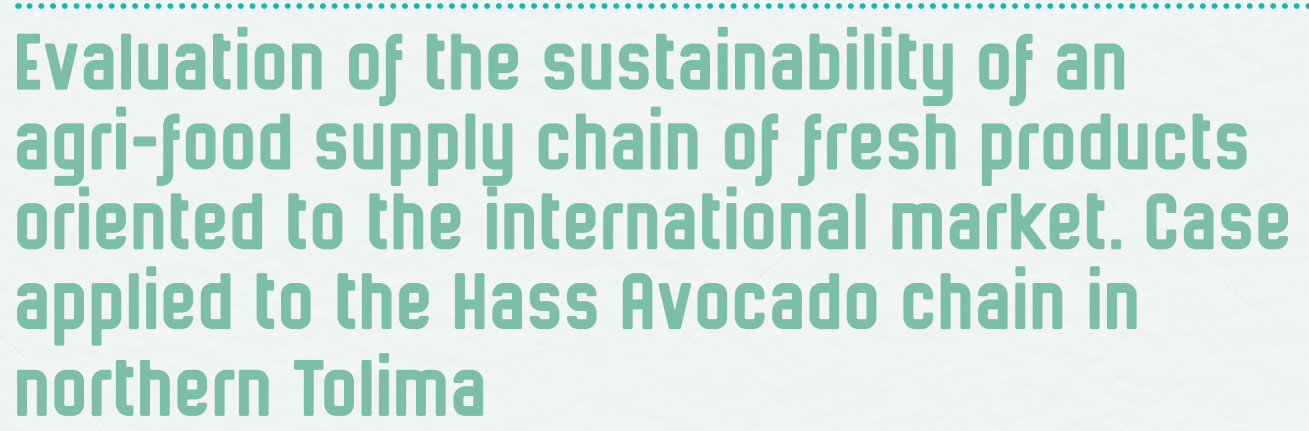

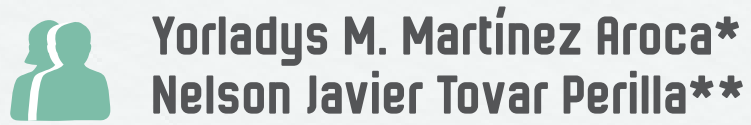

(- Recepción: 26/09/2019

(-) Aprobación: 27/11/2019

( Publicación: 19/12/2019

Para citar este artículo:

Martínez, Aroca, Y. M., \& Tovar Perilla, N. J. (2019). Evaluación de la sostenibilidad de una agrocadena de suministro de producto fresco orientada al mercado internacional. Caso aplicado a la cadena del Aguacate Hass en el norte del Tolima. Indagare, (7), 36-48. https://doi.org/10.35707/indagare/705

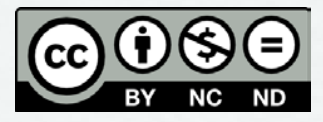

* Grupo de investigación NATURATU. Universidad de Ibagué. ORCID: 0000-0002-3581-988X. Correo electrónico: yorladys.martinez@unibague.edu.co

** Grupo de investigación GINNOVA. Universidad de Ibagué. ORCID: 0000-0001-6330-4181. Correo electrónico: nelson. tovar@unibague.edu.co 


\title{
Resumen
}

La internacionalización de productos frescos implica un gran reto sobre las cadenas de suministro de agroalimentos. Dadas sus características particulares, sus variables ocasionan que su estudio no sea una tarea fácil, como la perecibilidad de los productos, los altos porcentajes de pérdidas, la estacionalidad y variabilidad de la demanda y los precios, la ubicación de los productores y de los centros de acopio y comercialización, la poca disponibilidad de medios de transporte eficientes, los tratados de libre comercio y la aplicación de nuevas normas fitosanitarias para la exportación.

Uno de los primeros pasos en esta dirección, es comprender las interacciones y variables que determinan el funcionamiento de cada uno de los actores que conforman estas cadenas de suministro de agroalimentos. Este estudio presenta un modelo con el cual se recrea bajo ambiente virtual, la estructura y lazos de interacción presentes en la agrocadena del aguacate Hass en el norte del Tolima. El abordaje se realizó bajo el concepto de agrocadenas sostenibles, en el cual se toman en cuenta variables económicas, sociales y ambientales, utilizando el enfoque de dinámica de sistemas. Este proyecto pretende estudiar de manera prospectiva la sostenibilidad en la agrocadena del aguacate Hass en el norte del Tolima en su proceso de internacionalización.

\begin{abstract}
The internationalization of fresh products implies a great challenge on agri-food supply chains. Given its particular characteristics, its variables cause that studying them might not be an easy task to do, for instance: perishability of products; high percentages of losses; seasonality and variability of demand and prices; location of producers, collection and marketing centers; lack of availability of efficient means of transport; free trade agreements; and operation of new phytosanitary standards for export.

One of the first steps in this direction is to understand the interactions and variables that determine the functioning of each of the actors that make up these agri-food supply chains. This study introduces a model which through a virtual environment simulates structure and interaction bonds present in the Hass Avocado agri-food chain in northern Tolima. The approach was carried out under the concept of sustainable agri-food chains, in which economic, social and environmental variables are taken into account, using the systems dynamics approach. This project aims to study prospectively the sustainability in the Hass Avocado agri-food chain in northern Tolima in its internationalization process.
\end{abstract}


Palabras claves: Cadena de suministro de alimentos, aguacate, simulación, sostenibilidad, dinámica de sistemas.

Key words: Agri-food supply chain, avocado, simulation, sustainability, system dynamics.

\section{Introducción}

En las cadenas de suministro de alimentos mundiales se evidencian diversas problemáticas ambientales, sociales y económicas en las áreas dedicadas a la producción, por el uso inadecuado de prácticas de cultivo y poscosecha. Los principales problemas abarcan: a) la presencia de altos niveles de pobreza y desnutrición en buena parte de las zonas de producción ubicadas en los países subdesarrollados y en desarrollo, b) el surgimiento de crisis sanitarias de escala mundial originadas por el mal manejo de alimentos, c) la incorporación de nuevas tecnologías que han traído consigo la presencia y comercialización masiva de alimentos genéticamente modificados de los que aún no se tiene certeza de los efectos secundarios que pueden tener en su consumo humano, d) el deterioro presente en los ecosistemas que contienen las tierras aptas para la actividad agrícola, e) el cambio climático y la reducción significativa de la biodiversidad y las fuentes de agua potable por causa del desarrollo de actividades productivas (Srivastava, Singh, Tripathi, \& Raghubanshi, 2016), f) los altos porcentajes de pérdidas y desperdicios en las cadenas de alimentos, principalmente desde la poscosecha (FAO, 2012) y, g) el surgimiento de nuevas reglas de mercado para el intercambio y la trazabilidad de alimentos a nivel internacional (Aung \& Chang, 2014; Pires, et al., 2016).

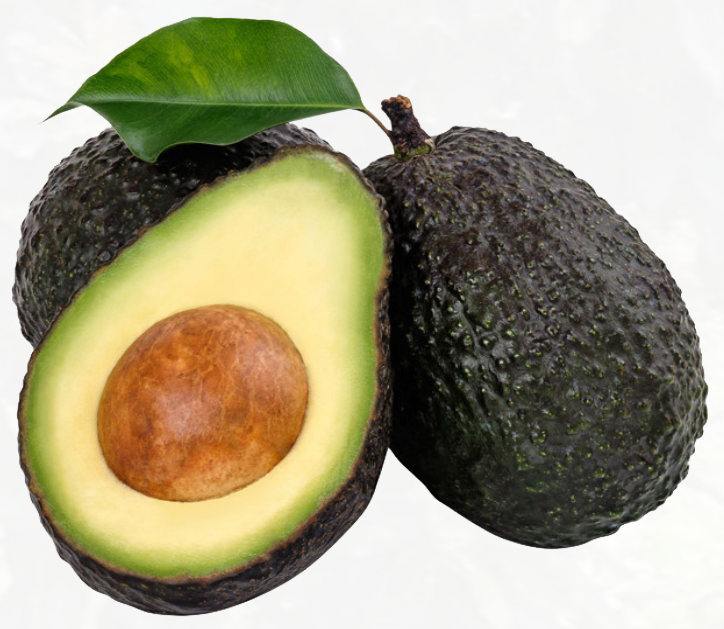


Para intentar resolver estas problemáticas presentes en las zonas de producción, se desarrollaron propuestas de modelos para el mejoramiento y la optimización de las cadenas de suministro de alimentos, dichas propuestas se diferencian por el alcance que tienen, las herramientas utilizadas y el ámbito de aplicación (Ahumada \& Villalobos, 2009). El alcance de los modelos ha sido principalmente estratégico (largo plazo) y táctico (mediano plazo). Las herramientas utilizadas son de naturaleza determinística, tales como la programación lineal, dinámica, mixta o meta; así como herramientas de naturaleza estocástica, entre ellas la programación estocástica y la simulación. La aplicación de estos modelos ha tenido lugar para cadenas de productos perecederos y no perecederos.

Diferentes autores desarrollaron modelos para el mejoramiento de las cadenas de suministro de alimentos, entre los aportes más relevantes encontramos el modelo para la planeación táctica de la operación y distribución de las cadenas de suministro de alimentos bajo condiciones de incertidumbre (Ahumada, Villalobos, \& Mason, 2012); modelo para analizar el proceso de toma de decisiones en las cadenas de suministro de alimentos (Tsolakis, Keramydas, Toka, Aidonis, \& Lakovou, 2014), modelo para la inclusión de los criterios de seguridad y calidad en la operación de las cadenas de suministro de alimentos (Aung \& Chang, 2014); modelo para evaluar el desempeño de cadenas de valor (Da Silva \& De Souza, 2007; Donovan, Cunha, Franzel, Gyau, \& Mithöfer, 2013; Bellù, 2013).

Otras herramientas aplicadas para el análisis de las cadenas de suministro en años recientes son optimización matemática (Shanthikumar, Yao, \& Zijm, 2003), el análisis envolvente de datos, la simulación discreta (Cimino, Longo, \& Mirabelli, 2010; AboHamad \& Arisha, 2011) y la simulación dinámica (Ramanathan \& Ramanathan, 2014). Esta última herramienta específicamente fue utilizada para analizar las cadenas de suministro de alimentos, en temas tan diversos como las relaciones entre los actores (Kumar \& Nigmatullin, 2011), la toma de decisiones estratégicas (Georgiadis, Vlachos, \& Lakovou, 2005), los impactos ex-ante de la implementación de políticas gubernamentales (Benson, 2009), y la sostenibilidad y calidad de los alimentos (Van der Vorst, Tromp, \& Van der Zee, 2009).

Colombia no es ajena a la problemática presentada anteriormente. En el país se pierden cerca de 9,76 millones de toneladas de alimentos al año, equivalentes al $34 \%$ frente al total producido; de esta cifra, cerca de 6,1 millones de toneladas corresponden a frutas y verduras, principalmente por el inadecuado manejo de estos productos desde la etapa de poscosecha (DNP, 2016). A pesar de las problemáticas en el sector agrícola en el país, el Tolima le ha apostado al desarrollo de productos agrícolas como el aguacate Hass, 


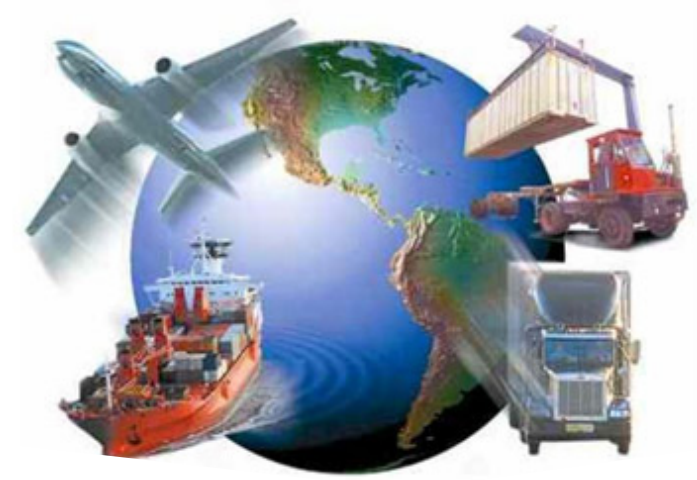

en el cual se ha logrado un potencial de crecimiento de ventas en el exterior superior al $200 \%$ en los últimos años, siendo los principales destinos los Países Bajos, seguido por Reino Unido y España (Ministerio de Agricultura, 2018).

No obstante, el potencial crecimiento de la cadena del aguacate Hass en el Tolima, presenta problemas que afectan su productividad y competitividad, tales como: las inequidades en el mercadeo, el uso de vehículos inadecuados para el transporte de los productos, el uso impropio de empaques para almacenamiento temporal en finca, incorrectas condiciones de almacenamiento en fincas y en centros de acopio, limitado uso de las buenas prácticas agrícolas y logísticas para frutas, la ausencia de herramientas informáticas y procesos estandarizados que ofrezcan trazabilidad en la operación logística de la cadena 'aguas abajo' en su cadena de valor, y bajo nivel de cooperación y asociación efectiva entre los actores (Universidad de Ibagué, 2016).

Por lo anterior, se han formulado proyectos de investigación y de transferencia tecnológica que permitirán mitigar los problemas presentes en la agrocadena del aguacate Hass en el norte del Tolima, entre ellos se puede mencionar el proyecto: "Diseño e implementación, a nivel piloto, de un modelo de gestión de cadena de suministro sustentable para la agrocadena del aguacate Hass en el norte del Tolima, con el fin de apoyar su proceso de internacionalización" financiado por la Universidad de Ibagué, y el proyecto: “Transferencia tecnológica para la optimización operacional de la agrocadena del aguacate en el Tolima como apoyo a su proceso de internacionalización” financiado por la Gobernación del Tolima y que hace parte del macroproyecto: "Desarrollo de ventajas competitivas mediante actividades $\mathrm{I}+\mathrm{D}+\mathrm{i}$ en ocho cadenas del sector agropecuario en el departamento del Tolima”.

En este artículo se presentan algunos avances logrados hasta el momento, relacionados con la evaluación de la sostenibilidad de una agrocadena de suministro de producto en fresco, orientada al mercado internacional mediante un modelo de simulación y validación de su funcionalidad en la agrocadena del aguacate Hass originaria en el norte del Tolima. 


\section{Materiales y métodos}

El proyecto se realiza con base en la metodología de investigación científica, propia de estudios de naturaleza descriptiva-propositiva, siguiendo un enfoque deductivo. El objeto de estudio son los actores de la agrocadena del aguacate Hass en el norte del Tolima.

Para el desarrollo del proyecto, inicialmente se determinarán las características de operación de una cadena de suministro de producto fresco orientada al mercado internacional, para ello se realizarán entrevistas, encuestas y visitas técnicas a diferentes actores de la cadena (productores, transformadores, transportadores y comercializadores); posteriormente, con los datos, se realizará el análisis estadístico descriptivo y se identificarán aquellos flujos que no permiten la operación eficiente de la agrocadena del aguacate Hass para su posicionamiento en mercados internacionales.

Luego, se procederá a evaluar el desempeño técnico de la cadena de suministro del aguacate Hass en el norte del Tolima, mediante herramientas de simulación. Para ello, en primer lugar se realizará una estandarización de los procedimientos por medio de una recopilación de información de los diferentes procesos, materias primas empleadas y tiempos para cada proceso. Para la construcción del modelo de simulación, se desarrollará un modelo mediante el enfoque de dinámica de sistemas, dado que una cadena de suministro de agroalimentos es un sistema en el que intervienen diversos actores con múltiples relaciones y flujos (Figura 1), lo cual se puede considerar como un sistema complejo (Sterman, 2000).

Figura 1. Modelo de simulación - Modelo conceptual

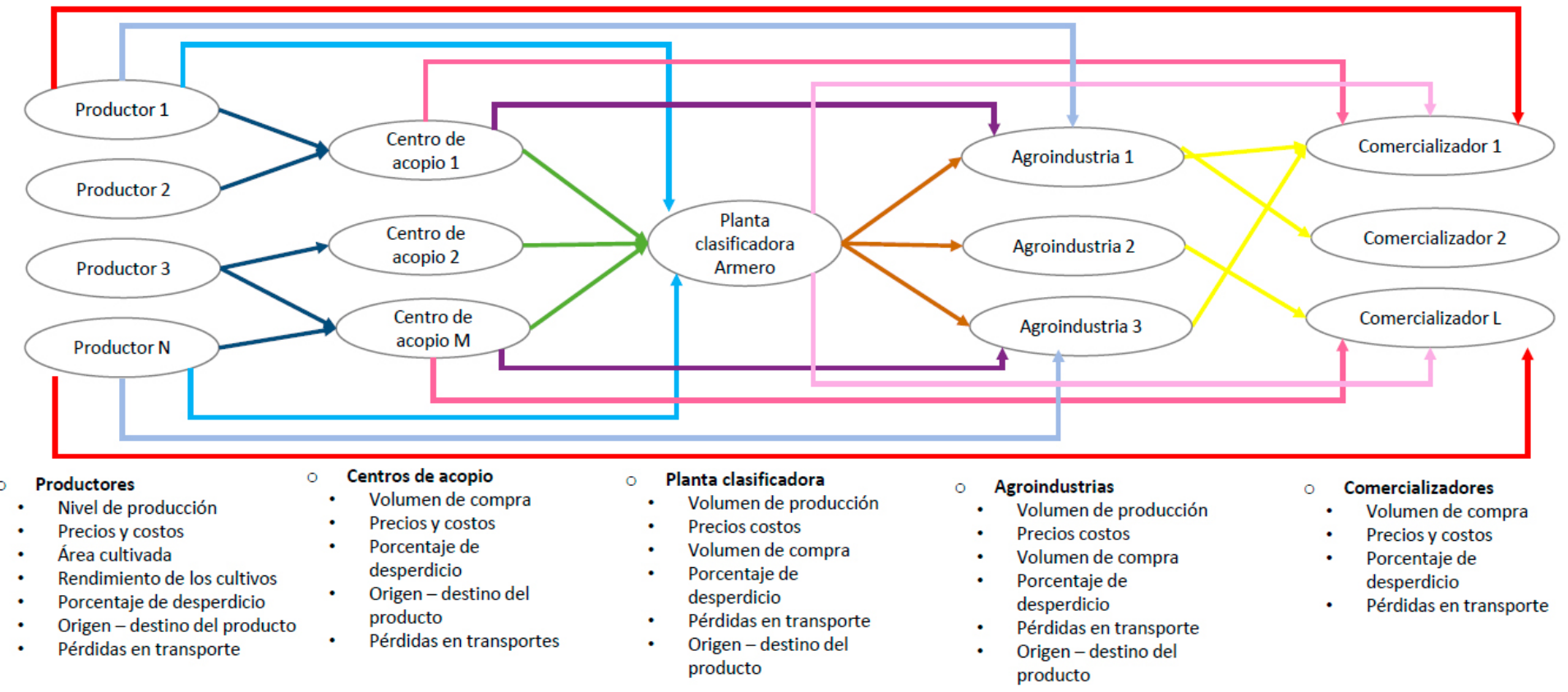

Fuente: Elaboración propia. 
A partir de lo anterior, se desarrollarán escenarios futuros para el proceso de internacionalización del aguacate Hass del norte del Tolima, para ello se tendrán en cuenta los resultados del modelo de simulación alimentado con la información de las variables propias de la agrocadena.

Por último, se diseñarán acciones de mejora, orientadas a los procesos de internacionalización, por medio de la selección de los escenarios más convenientes para la agrocadena del aguacate Hass en el norte del Tolima.

\section{Resultados preliminares}

Los resultados parciales obtenidos hasta el momento, en el desarrollo del proyecto, están relacionados con la construcción del modelo de simulación y del enfoque para evaluar la sostenibilidad de una cadena de suministro de agroalimentos, el cual analiza factores económicos, ambientales y sociales (Figura 2).

Figura 2. Enfoque de abordaje: Pilares de la sostenibilidad
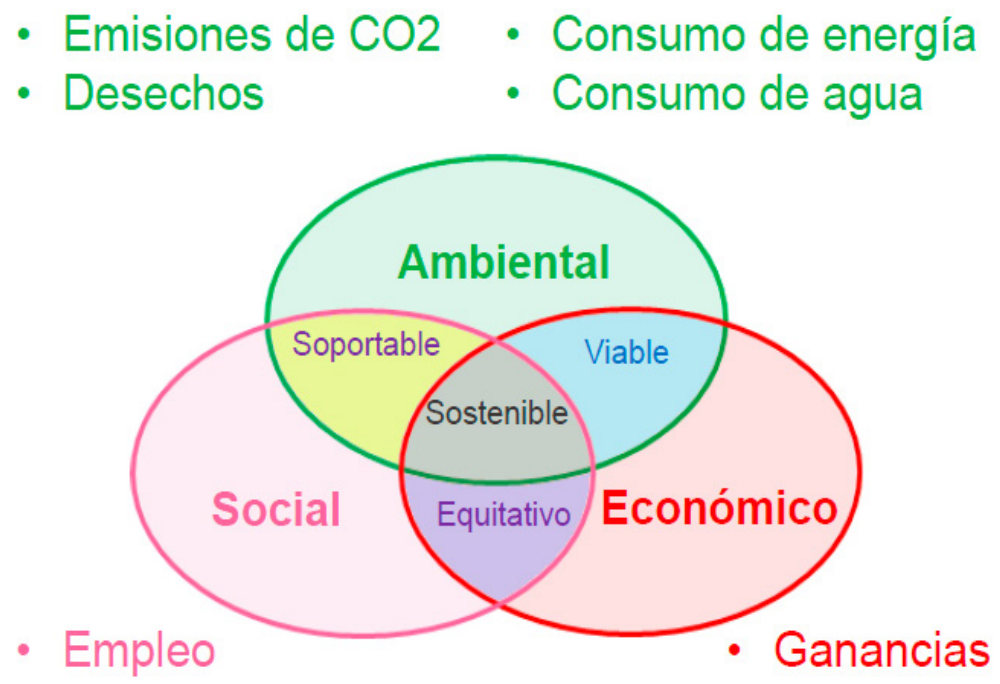

Fuente: Elaboración propia basada en ONU.

Adicional a lo anterior, dadas las características que presentan los actores de la cadena y las relaciones que existen entre ellos, el modelo de simulación general (Figura 3) está constituido por seis submodelos; los cinco primeros representan la dinámica e interacción que se mantiene en los diferentes actores de la agrocadena del aguacate Hass en 
Divulgación científica

el norte del Tolima (productores, centros de acopio, planta clasificadora, agroindustrias y comercializadores), al tener en cuenta que su construcción hace referencia a las variables propias de cada actor.

Figura 3. Modelo de simulación general de la agrocadena del aguacate Hass en el norte del Tolima
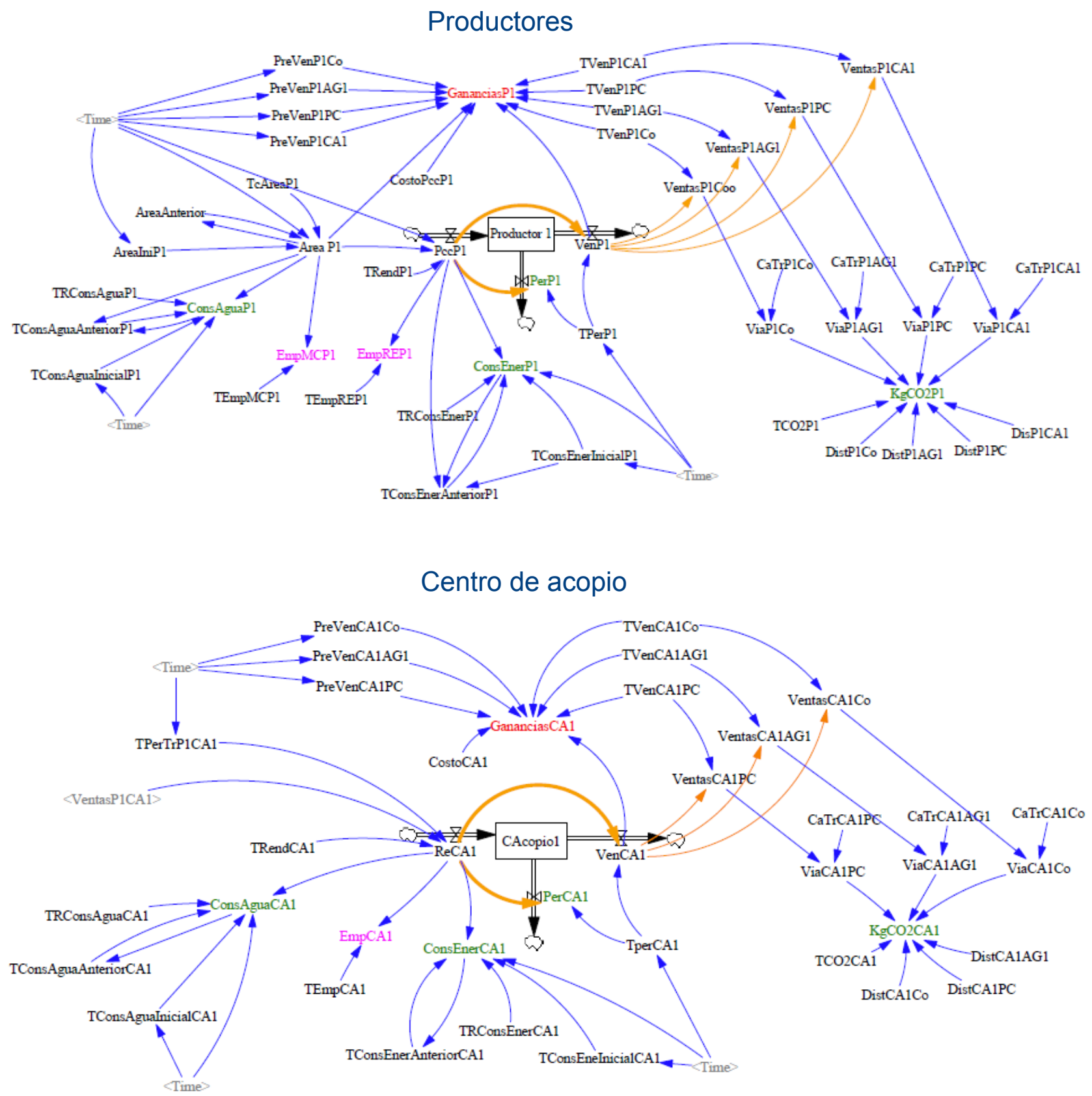


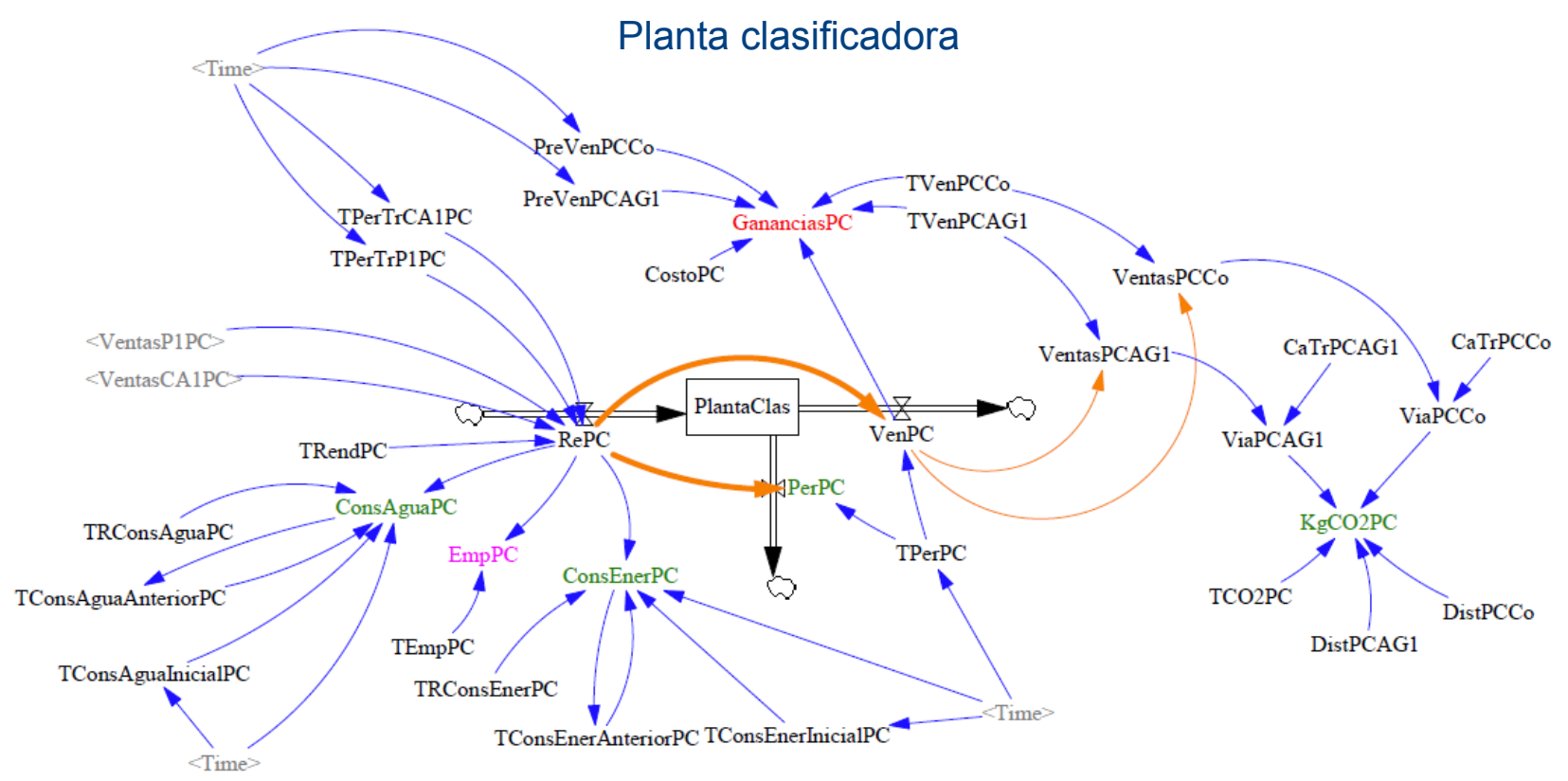

\section{Agroindustrias}

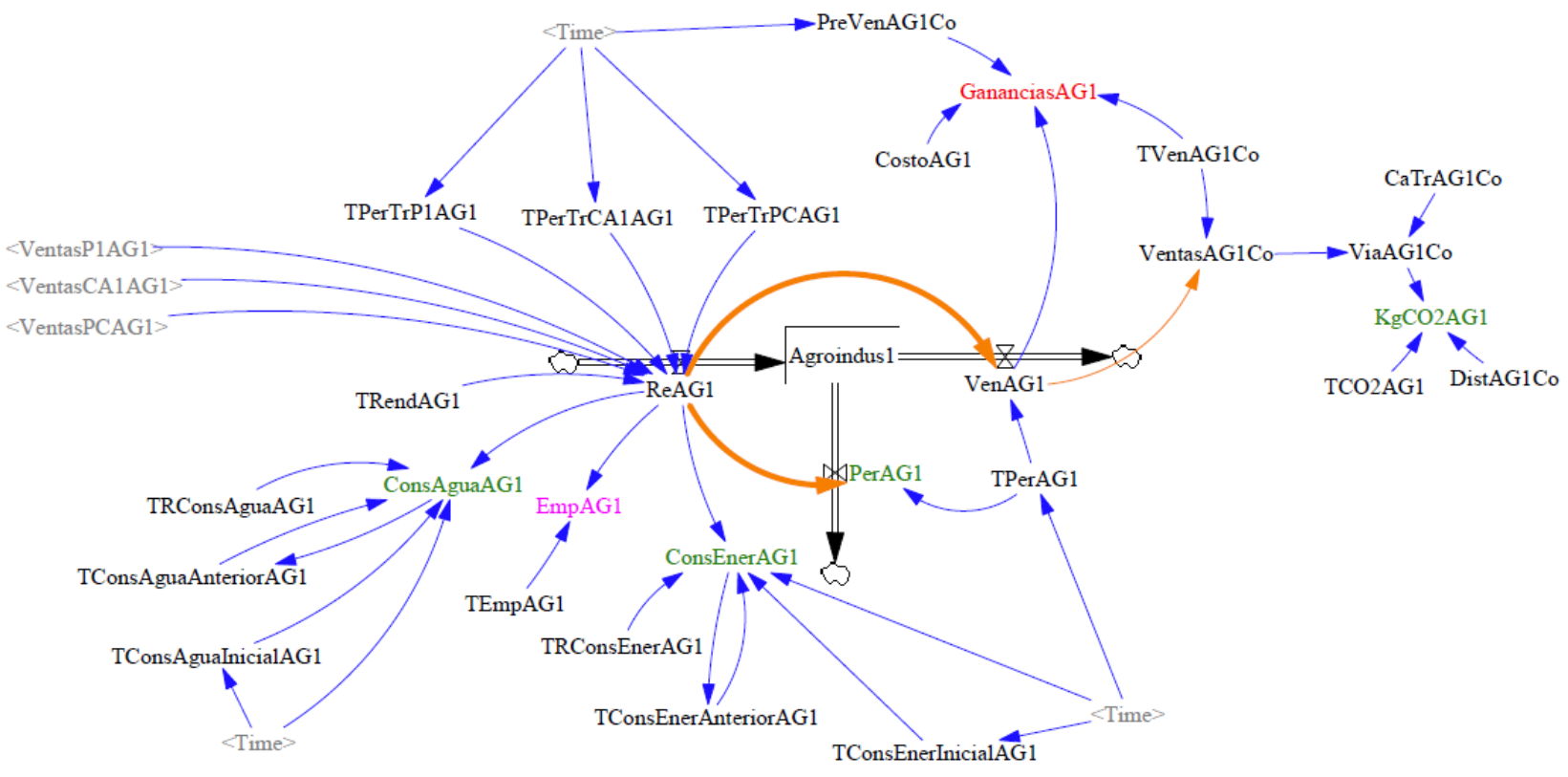

Fuente: Elaboración propia en software Vensim. 


\section{Comercializadores}

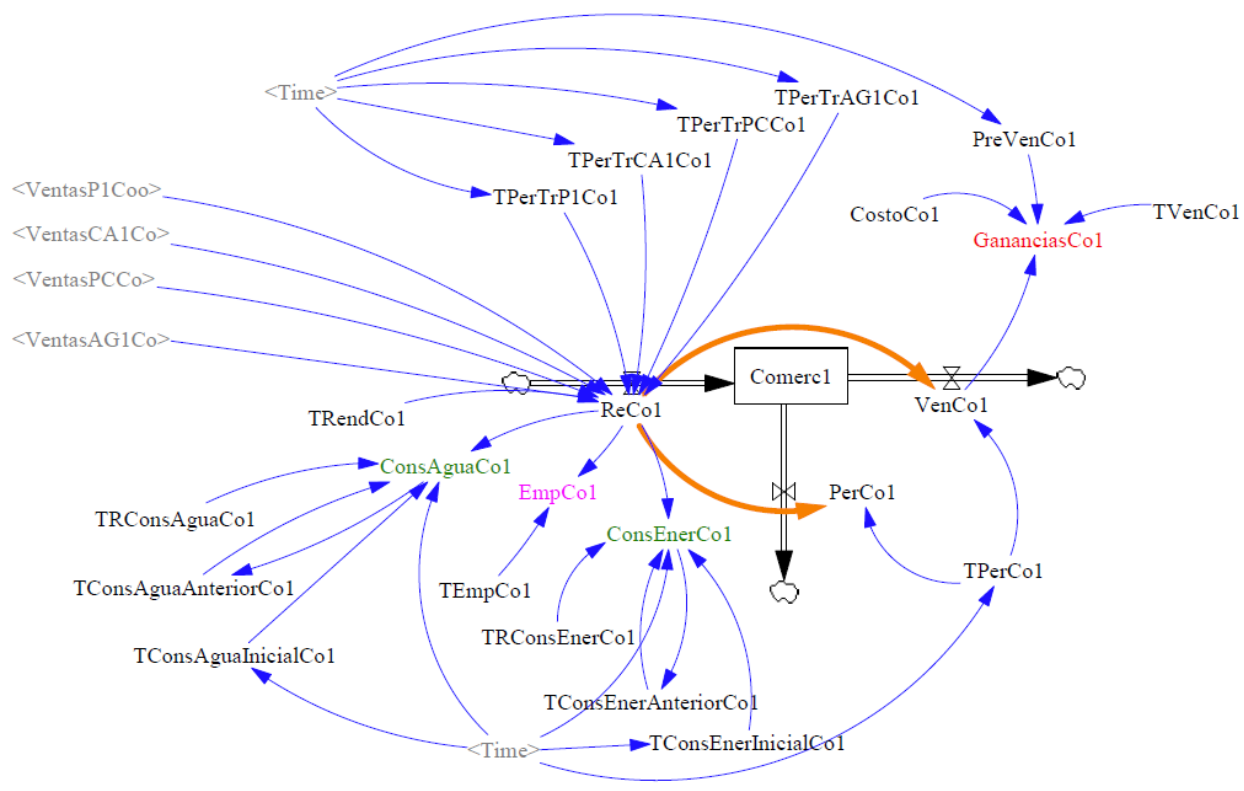

\section{Medidas de desempeño}

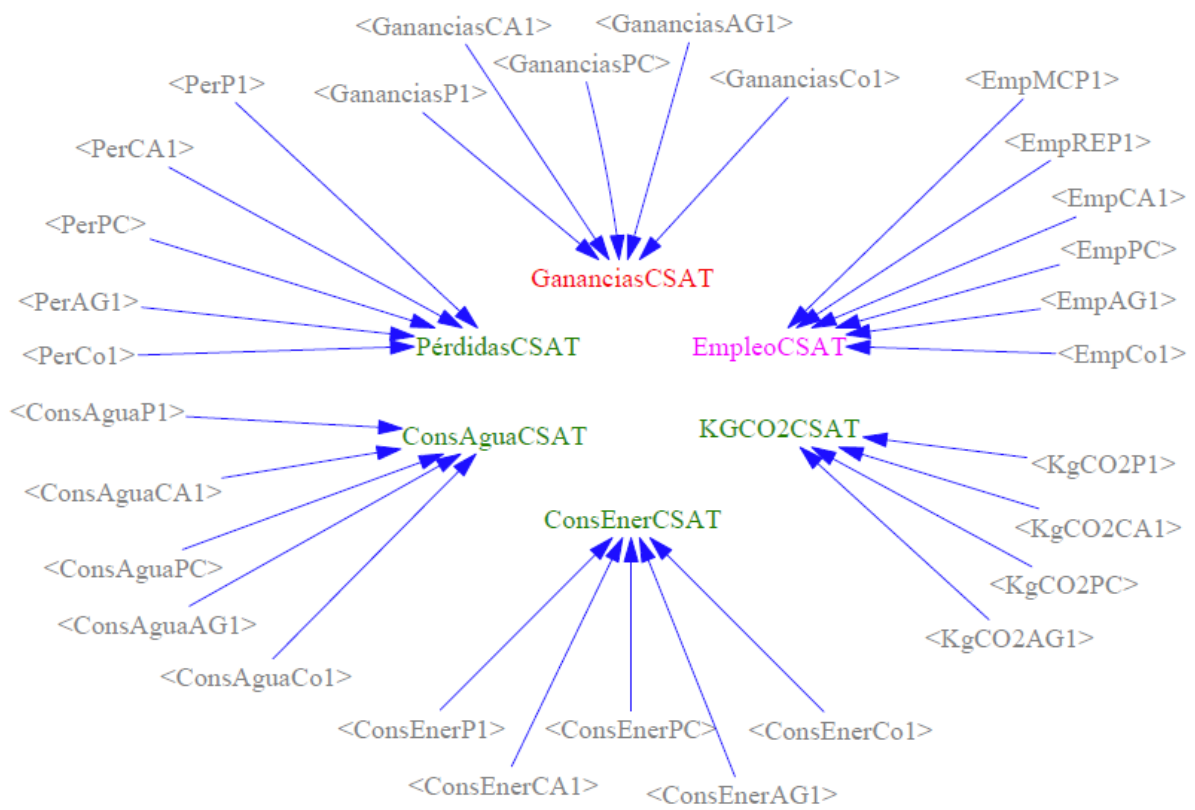

Fuente: Elaboración propia en software Vensim. 
El sexto submodelo hace referencia a las medidas de desempeño que se tendrán en cuenta para la evaluación de la sostenibilidad de la agrocadena del aguacate Hass en el norte del Tolima, dichas medidas están constituidas por las variables de salida de cada submodelo asociado a cada uno de los actores de la agrocadena del aguacate Hass en el norte del Tolima (Figura 3, medidas de desempeño).

\section{Potencial uso}

Las acciones orientadas en la mejora e internacionalización de algunas de las agrocadenas en el Tolima requieren del desarrollo de estudios de carácter científico-tecnológico, que ofrezcan información pertinente y oportuna a los tomadores de decisiones, para contribuir a su buen funcionamiento a largo plazo, es por ello que estos resultados preliminares que arroja esta investigación constituyen un aporte práctico y metodológico para futuras investigaciones, las cuales tengan como características diversas relaciones e interacciones entre actores, en miras de la posible internacionalización.

\section{Ficha técnica del proyecto}

Título del proyecto: Evaluación de la sostenibilidad de una agrocadena de suministro de producto fresco orientada al mercado internacional. Caso aplicado a la cadena del aguacate Hass en el norte del Tolima.

PRIT: Industria de servicios de alto valor agregado y de talla internacional.

Código del proyecto: 19-521-TES

Investigador principal: Yorladys Martínez Aroca.

Duración del proyecto: 12 meses.

\section{Referencias}

Abo-Hamad, W., \& Arisha, A. (2011). Simulation Optimisation Methods in Supply Chain Applications: A review. Irish Journal of Management, 30 (2), 95-124. Recuperado de https://arrow.tudublin.ie/ $\mathrm{cgi} /$ viewcontent.cgi?article $=1113 \&$ context $=$ buschmarart

Ahumada, O., \& Villalobos, J. R. (2009). Application of planning models in the agri-food supply chain: A review. European Journal of Operational Research, 196 (1), 1-20. https://doi.org/10.1016/j. ejor.2008.02.014

Ahumada, O., Villalobos, J. R. \& Mason, A. N. (2012). Tactical planning of the production and distribution of fresh agricultural products under uncertainty. Agricultural Systems, 112, 17-26. https://doi.org/10.1016/j.agsy.2012.06.002 
Aung, M. M., \& Chang. Y. S. (2014). Traceability in a food supply chain: Safety and quality perspectives. Food Control, 39(1), 172-184. https://doi.org/10.1016/j.foodcont.2013.11.007

Bellù, L.G. (2013). Value Chain Analysis for Policy Making: Methodological Guidelines and Country Cases for a Quantitative Approach (EASYPol Series 129). Recuperado del sitio de internet de FAO http://www.fao.org/3/a-at511e.pdf

Benson, K. (2009). Ex-ante impact assessment of livestock interventions in crop-livestock systems in northern Afghanistan (Master of Science Thesis, Cornell University, Faculty of the Graduate School, Ithaca, NY). Recuperado de https://hdl.handle.net/1813/11535

Cimino, A., Longo, F., \& Mirabelli, G. (2010). A general simulation framework for supply chain modeling: State of the art and case study. International Journal of Computer Science Issues, 7 (2), 1-9. Recuperado de https://www.researchgate.net/publication/43225357_A_General_ Simulation_Framework_for_Supply_Chain_Modeling_State_of_the_Art_and_Case_Study

Da Silva, C. \& De Souza, H. (2007). Guidelines for rapid appraisals of agrifood chain performance in developing countries. Roma, Italy: FAO. Recuperado de http://www.fao.org/3/a1475e/a1475e00.htm

Departamento Nacional de Planeación (DNP) (2016). Pérdidas y desperdicios de alimentos en Colombia. Recuperado de https://mrv.dnp.gov.co/Documentos\%20de\%20Interes/Perdida_y_Desperdicio_ de_Alimentos_en_colombia.pdf

Donovan, J., Cunha, M., Franzel, S., Gyau, A., \& Mithöfer, D. (2013). Guides for Value Chain Development: A Comparative Review. Wageningen, The Netherlands: CTA \& World Agroforestry Centre. Recuperado de https://hdl.handle.net/10568/75498

FAO (2012). Pérdidas y desperdicio de alimentos en el mundo - Alcance, causas y prevención. Recuperado de http://www.fao.org/3/a-i2697s.pdf

Georgiadis, P., Vlachos, D., \& Lakovou, E. (2005). A system dynamics modeling framework for the strategic supply chain management of food chains. Journal of Food Engineering, 70 (3), 351-364. https://doi.org/10.1016/j.jfoodeng.2004.06.030

Kumar, S., \& Nigmatullin, A. (2011). A system dynamics analysis of food supply chains - Case study with non-perishable products. Simulation Modelling Practice and Theory, 19 (10), 2151-2168. https://doi.org/10.1016/j.simpat.2011.06.006

Ministerio de Agricultura. (2018). Estadísticas. Inicio. Recuperado de http://www.agronet.gov.co/ estadistica/Paginas/default.aspx

Pires, G., Abrahão, G., Brumatti, L., Oliveira, L., Costa, M., Liddicoat, S., \& Ladle, R. (2016). Increased climate risk in Brazilian double cropping agriculture systems: Implications for land use in Northern Brazil. Agricultural and Forest Meteorology, 228-229, 286-298. https://doi. org/10.1016/j.agrformet.2016.07.005

Ramanathan, U., \& Ramanathan, R. (2014). Supply chain strategies, issues and models. London, UK: Springer.

Shanthikumar, J. G., Yao, D. D., \& Zijm, W. H. (Eds.). (2003). Stochastic modeling and optimization of manufacturing systems and supply chains. International series in operations research \& management science, 63. New York: Kluwer Academic Publishers.

Srivastava, P., Singh, R., Tripathi, S., \& Raghubanshi, A. S. (2016). An urgent need for sustainable thinking in agriculture - An Indian scenario. Ecological Indicators, 67, 611-622. https://doi. org/10.1016/j.ecolind.2016.03.015 
Sterman, J. D. (2000). Business Dynamics: Systems Thinking and Modeling for a Complex World. Recuperado de https://www.researchgate.net/publication/44827001_Business_Dynamics_ System_Thinking_and_Modeling_for_a_Complex_World

Tsolakis, N. K., Keramydas, C. A., Toka A. K., Aidonis, D.A., \& Lakovou, E F. (2014). Agrifood supply chain management: A comprehensive hierarchical decision-making framework and a critical taxonomy. Biosystems Engineering, 120, 47-64. https://doi.org/10.1016/j.biosystemseng.2013.10.014

Universidad de Ibagué (2016). Convenio Especial de Cooperación No 1032-2013. “Diseñar e implementar alternativas logísticas orientadas al mejoramiento competitivo y el desarrollo tecnológico de la cadena hortofrutícola del Tolima” Ibagué, Colombia: Universidad de Ibagué-Gobernación del Tolima.

Van der Vorst, J., Tromp, S., \& Van der Zee, D. (2009). Simulation modelling for food supply chain redesign; integrated decision making on product quality, sustainability and logistics. International Journal of Production Research, 47 (23), 6611-6631. https://doi.org/10.1080/00207540802356747 